\title{
Modeling studies for designing transcranial direct current stimulation protocol in Alzheimer's disease
}

\section{Shirin Mahdavi, Fatemeh Yavari, Shahriar Gharibzadeh* and Farzad Towhidkhah}

Department of Biomedical Engineering, Amirkabir University of Technology, Tehran, Iran

${ }^{*}$ Correspondence: gharibzadeh@aut.ac.ir

Edited by:

Tobias Alecio Mattei, Ohio State University, USA

Reviewed by:

Al-Rahim Abbasali Tailor, The Ohio State University Wexner Medical Center, USA

Keywords: brain stimulation, transcranial direct current stimulation (tDCS), computational modeling, finite element model, human head model, Alzheimer's disease

Transcranial direct current stimulation (tDCS) has been proposed as a technique for brain activity modulation. In this technique, a weak current (usually $1-2 \mathrm{~mA}$ ) is delivered to scalp through two sponge electrodes. There are two types of tDCS stimulation: cathodal and anodal, which inhibit and facilitate neuronal activity, respectively (Hansen, 2012).

tDCS has been shown to be effective in Alzheimer's disease (AD). Several studies have revealed that tDCS application can improve memory performance in Alzheimer's patients (APs) (Ferrucci et al., 2008; Boggio et al., 2009, 2012). For example, results of a single session tDCS study (Ferrucci et al., 2008) revealed that anodal/cathodal tDCS significantly enhanced/worsened word recognition in $\mathrm{AD}$ patients. In another study, application of anodal stimulation over DLPFC of APs has led to recognition memory improvement in a visual memory task (Boggio et al., 2009). These effects seem to be persistent, as in a multi-session tDCS study (Boggio et al., 2012), improvement in patients' visual recognition lasted for 4 weeks.

Current pathway through brain plays a key role in the observed effects. Currently, modeling studies provide the only way for determining the pattern of current flow during tDCS. In recent years, finite element modeling has been suggested as a reliable and helpful tool in clinical therapeutic applications (Bikson et al., 2012).

A critical issue which is required to be considered in modeling studies is the inter-individual anatomical variations. A modeling study has shown the profound role of individual cortical morphology in determination of current flow distribution for healthy people (Datta et al., 2012). Also the impact of pathologic anatomy (skull defects and lesions) on modulation of current flow has been examined in some previous studies (Datta et al., 2010, 2011). Specifically, in AD loss of neuronal structures and synaptic damages result in cortex shrinkage and ventricular enlargement (Frisoni et al., 2010). This changes the volume of CSF- referred as "super highway" for current flow- and therefore can significantly alters current pathway in these patients' head compared to healthy subjects (Bikson et al., 2012). These studies suggest that it is not precise to determine the dosage of applied current only based on healthy human modeling or clinical trial outcomes.

We hypothesize that change in cortical thickness due to brain atrophy has significant effects on current flow pattern. These anatomical alterations may shift the stimulated areas and peak current density location in head. They may even alter the expected results from tDCS application.

We suggest that cortical thickness is required to be considered in modeling studies to obtain more precise pattern of current flow in head and the stimulated brain regions. Specifically, AD affects differently on each patient's brain structure. We suggest developing individualized models based on each patient's MRI data. These models can be used by clinicians to find the optimal electrode montage and current amplitude for each patient.

Using Individual-based models for designing clinical protocols could provide us with better interpretation of the results.

\section{REFERENCES}

Bikson, M., Rahman, A., Datta, A., Fregni, F., and Merabet, L. (2012). High-resolution modeling assisted design of customized and individualized transcranial direct current stimulation protocols. Neuromodulation 15, 306-315. doi: 10.1111/j.1525-1403.2012. 00481.x

Boggio, P. S., Ferrucci, R., Mameli, F., Martins, D., Martins, O., Vergari, M., et al. (2012). Prolonged visual memory enhancement after direct current stimulation in Alzheimer's disease. Brain Stimul. 5, 223-230. doi: 10.1016/j.brs.2011. 06.006

Boggio, P. S., Khoury, L. P., Martins, D. C. Martins, O. E., De Macedo, E. C., and Fregni, F. (2009). Temporal cortex direct current stimulation enhances performance on a visual recognition memory task in Alzheimer disease. J. Neurol. Neurosurg. Psychiatry 80, 444-447. doi: 10.1136/jnnp.2007. 141853

Datta, A., Baker, J. M., Bikson, M., and Fridriksson, J. (2011). Individualized model predicts brain current flow during transcranial direct-current stimulation treatment in responsive stroke patient. Brain Stimul. 4, 169-174. doi: 10.1016/j.brs.2010. 11.001

Datta, A., Bikson, M., and Fregni, F. (2010). Transcranial direct current stimulation in patients with skull defects and skull plates: high-resolution computational FEM study of factors altering cortical current flow. Neuroimage 52, 1268-1278. doi: 10.1016/j.neuroimage.2010. 04.252

Datta, A., Truong, D., Minhas, P., Parra, L. C., and Bikson, M. (2012). Inter-individual variation during transcranial direct current stimulation and normalization of dose using MRI-derived computational models. Front. Psychiatry 3:91. doi: 10.3389/fpsyt.2012.00091

Ferrucci, R., Mameli, F., Guidi, I., MrakicSposta, S., Vergari, M., Marceglia, S., et al. (2008). Transcranial direct current stimulation improves recognition memory in Alzheimer disease. Neurology 71 
493-498. doi: 10.1212/01.wnl.0000317060. 43722.a3

Frisoni, G. B., Fox, N. C., Jack, C. R. Jr., Scheltens, P., and Thompson, P. M. (2010). The clinical use of structural MRI in Alzheimer disease. Nat. Rev. Neurol. 6, 67-77. doi: 10.1038/nrneurol. 2009. 215

Hansen, N. (2012). Action mechanisms of transcranial direct current stimulation in Alzheimer's disease and memory loss. Front. Psychiatry 3:48. doi: 10.3389/fpsyt.2012. 00048
Conflict of Interest Statement: The authors declare that the research was conducted in the absence of any commercial or financial relationships that could be construed as a potential conflict of interest.

Received: 30 January 2014; accepted: 27 June 2014; published online: 22 July 2014.

Citation: Mahdavi S, Yavari F, Gharibzadeh $S$ and Towhidkhah F (2014) Modeling studies for designing transcranial direct current stimulation protocol in Alzheimer's disease. Front. Comput. Neurosci. 8:72. doi: 10.3389/fncom.2014.00072
This article was submitted to the journal Frontiers in Computational Neuroscience.

Copyright (c) 2014 Mahdavi, Yavari, Gharibzadeh and Towhidkhah. This is an open-access article distributed under the terms of the Creative Commons Attribution License (CC BY). The use, distribution or reproduction in other forums is permitted, provided the original author(s) or licensor are credited and that the original publication in this journal is cited, in accordance with accepted academic practice. No use, distribution or reproduction is permitted which does not comply with these terms. 